\title{
ccereative
commons
}

ISSN 1855-3966 (printed edn.), ISSN 1855-3974 (electronic edn.)

\author{
ARS MATHEMATICA CONTEMPORANEA 16 (2019) 465-472 \\ https://doi.org/10.26493/1855-3974.1699.74e \\ (Also available at http://amc-journal.eu)
}

\section{Some extensions of optimal stopping with financial applications}

\author{
Mihael Perman * \\ Faculty of Mathematics and Physics, University of Ljubljana, \\ Jadranska 19, SI-1000 Ljubljana, Slovenia and \\ University of Primorska, Faculty of Mathematics, Natural Sciences and Information \\ Technologies, Glagoljaška 8, SI-6000 Koper, Slovenia \\ Ana Zalokar ${ }^{\dagger}$ \\ University of Primorska, Faculty of Mathematics, Natural Sciences and Information \\ Technologies, Glagoljaška 8, SI-6000 Koper, Slovenia and \\ University of Primorska, Andrej Marušič Institute, \\ Muzejski trg 2, SI-6000 Koper, Slovenia
}

Received 15 May 2018, accepted 19 June 2018, published online 22 February 2019

\begin{abstract}
Finite horizon optimal stopping problems for Markov chains are a well researched topic. Frequently they are phrased in terms of cost or return because many financial models are based on Markov chains. In this paper we will apply optimal stopping to certain random walks on binary trees motivated by insurance considerations. The results are direct extensions of known results but the implications for insurance are of interest.
\end{abstract}

Keywords: Optimal stopping for Markov chains, equity-linked life insurance with guarantees.

Math. Subj. Class.: 60G40, $91 B 30$

\section{Introduction}

Modern insurance regulation requires companies to apply market valuation to assets and liabilities. The value of assets can be determined directly from market prices, or through appropriate approximations using fair value methodology. For insurance liabilities, however, there is no regulated market to determine their value. The particular case that we

\footnotetext{
* This work is supported in part by the Slovenian Research Agency (research program P1-0285).

${ }^{\dagger}$ This work is supported in part by the Slovenian Research Agency (research program P1-0285 and Young Researchers Grant).

E-mail addresses: mihael.perman@fmf.uni-lj.si (Mihael Perman), ana.zalokar@iam.upr.si (Ana Zalokar)
} 
will be considering here are equity-linked life policies with guarantees. The policyholder invests her premium in an underlying fund managed by the insurance company. A typical example are long term pension saving products. In recent years there has been a tendency to attach guarantees such as a minimum return or a minimum death benefit guarantee to these investments which gives rise to new liabilities. In many cases guarantees can be interpreted as contingent claims on the underlying fund, for example guarantees in equity-linked products or complementary health policies with equalization schemes, see [10].

Nonnenmacher was one of the first authors to interpret guarantees as put options on the value on the underlying fund, [8] and [7]. Once a stochastic model for the dynamics of the fund value is formulated, the liabilities arising from guarantees can be valued using the methods to value derivative securities. Paper [6] considers equity-linked products as contingent claims on the value of the underlying asset but introduces mortality as an independent additional source of randomness. The assumption of independence is often made, see [5] for some implications. With this addition the model is no longer complete and the paper considers optimal hedging strategies that minimize the expected cost for the insurer.

In this paper we will present some extensions of optimal stopping rules motivated by financial questions in insurance. The proofs follow the steps of classical proofs but the formulation of the problems is slightly different. These results will then be applied to investigate relative merits of different ways an insurance company can hedge its liabilities. The models are simplified versions of reality but can shed some light on what strategies may lead to best results.

\section{Variations of optimal stopping rules}

The classical finite horizon optimal stopping problem for a general finite length inhomogeneous Markov chain $X_{0}, X_{1}, \ldots, X_{N}$ and general state space is to minimize the expression

$$
E\left[g\left(X_{\nu}\right)+\sum_{j=1}^{\nu-1} c\left(X_{j}\right)\right]
$$

where $\nu$ runs over all stopping times with respect to the filtration of the Markov chain, and $g$ and $c$ are given functions. For the sake of simplicity it will be assumed that $g$ and $c$ are bounded. For Markov chains it is enough to solve the problem assuming that $X_{0}=x$ for $x$ in the state space. Denote the value function $v_{N}$ by

$$
v_{N}(x)=\inf _{\{\nu: P(\nu \leq N)=1\}} E\left[g\left(X_{\nu}\right)+\sum_{j=1}^{\nu-1} c\left(X_{j}\right) \mid X_{0}=x\right]
$$

The dynamic programming equations for this problem are defined recursively as

$$
\begin{aligned}
V_{N}(x) & :=g(x) \\
V_{n}(x) & :=\min \left\{g(x), c(x)+E\left[V_{n+1}\left(X_{n+1}\right) \mid X_{n}=x\right]\right\}
\end{aligned}
$$

for $n=0,1, \ldots, N-1$. The solution to the stopping problem is given by

Theorem 2.1. The value function is given by $v_{N}(x)=V_{0}(x)$, and the optimal stopping time is given by

$$
\nu=\inf \left\{j \geq 0: V_{j}\left(X_{j}\right)=g\left(X_{j}\right)\right\} .
$$


See [9] for proofs.

The above stopping problem has many possible extensions and generalizations. For the financial application in this paper we will minimize the expression

$$
E\left[g_{\nu}\left(X_{1}, \ldots, X_{\nu}\right)+\sum_{j=1}^{\nu-1} c_{j}\left(X_{1}, \ldots, X_{j}\right)\right]
$$

for all stopping times $\nu \leq N$ for given functions $c_{1}, \ldots, c_{N}$ and $g_{1}, \ldots, g_{N}$. The dynamic programming equations in this more general setup are

$$
\begin{aligned}
& V_{N}\left(x_{0}, \ldots, x_{N}\right):=g_{N}\left(x_{0}, \ldots, x_{N}\right) \\
& V_{n}\left(x_{0}, \ldots, x_{n}\right):=\min \left\{g_{n}\left(x_{0}, \ldots, x_{n}\right),\right. \\
&\left.c_{n}\left(x_{0}, \ldots, x_{n}\right)+E\left[V_{n+1}\left(x_{0}, \ldots, x_{n}, X_{n+1}\right) \mid X_{n}=x_{n}\right]\right\}
\end{aligned}
$$

The optimal time is given by

$$
\nu_{N}=\inf \left\{j \geq 0: V_{j}\left(X_{0}, \ldots, X_{j}\right)=g_{j}\left(X_{0}, \ldots, X_{j}\right)\right\} .
$$

For the sake of completness we give the proof of this more general theorem. Define

$$
Z_{n}=\sum_{j=0}^{n-1} c_{j}\left(X_{0}, \ldots, X_{j}\right)+V_{n}\left(X_{0}, \ldots, X_{n}\right)
$$

for $j=0,1, \ldots, N$. With this definition we have

Theorem 2.2. The process $\left(Z_{n}\right)_{0 \leq n \leq N}$ is a submartingale with respect to the filtration of the Markov chain.

Proof. Denote $\mathcal{F}_{n}=\sigma\left(X_{0}, \ldots, X_{n}\right)$ for $1 \leq n \leq N$. We compute

$$
\begin{aligned}
E\left[Z_{n+1} \mid \mathcal{F}_{n}\right] & =\sum_{j=0}^{n} c_{j}\left(X_{0}, \ldots, X_{j}\right)+E\left[V_{n+1}\left(X_{0}, \ldots, X_{n+1}\right) \mid \mathcal{F}_{n}\right] \\
& \geq \sum_{j=0}^{n-1} c_{j}\left(X_{0}, \ldots, X_{j}\right)+V_{n}\left(X_{0}, \ldots, X_{n}\right) \\
& =Z_{n} .
\end{aligned}
$$

Theorem 2.3. For the time $\nu_{N}$ defined in (2.6) the expression (2.5) attains its minimum which equals $E\left(V_{0}\left(X_{0}\right)\right)$.

Proof. By Theorem 2.2

$$
E\left[Z_{\nu}\right] \geq E\left[Z_{0}\right]=E\left[V_{0}\left(X_{0}\right)\right]
$$

for all stopping times $\nu$. By definition we have

$$
E\left[V_{0}\left(X_{0}\right)\right] \leq E\left[Z_{\nu}\right] \leq E\left[\sum_{j=0}^{\nu-1} c_{j}\left(X_{0}, \ldots, X_{j}\right)+g_{\nu}\left(X_{0}, \ldots, X_{\nu}\right)\right]
$$


Replacing $\nu$ by $\nu_{N}$ we have

$$
\begin{aligned}
& E\left[Z_{\nu_{N} \wedge(n+1)} \mid \mathcal{F}_{n}\right] \\
& =1\left(\nu_{N} \leq n\right) Z_{\nu_{N}}+1\left(\nu_{N} \geq n+1\right) E\left[Z_{n+1} \mid \mathcal{F}_{n}\right] \\
& =1\left(\nu_{N} \leq n\right) Z_{\nu_{N}} \\
& \quad+1\left(\nu_{N} \geq n+1\right)\left[\sum_{j=1}^{n} c_{j}\left(X_{0}, \ldots, X_{j}\right)+E\left[V_{n+1}\left(X_{0}, \ldots, X_{n+1} \mid X_{0}, \ldots, X_{n}\right]\right]\right. \\
& =1\left(\nu_{N} \leq n\right) Z_{\nu_{N}}+1\left(\nu_{N} \geq n+1\right)\left[\sum_{j=1}^{n-1} c_{j}\left(X_{0}, \ldots, X_{j}\right)+V_{n}\left(X_{0}, \ldots, X_{n}\right)\right] \\
& =1\left(\nu_{N} \leq n\right) Z_{\nu_{N}}+1\left(\nu_{N} \geq n+1\right) Z_{n} \\
& =Z_{\nu_{N} \wedge n} .
\end{aligned}
$$

It follows that

$$
E\left[V_{0}\left(X_{0}\right)\right]=E\left[Z_{\nu_{N} \wedge 1}\right]=\cdots=E\left[Z_{\nu_{N} \wedge N}\right] .
$$

By (2.10) - (2.12) the minimum $E\left[V_{0}\left(X_{0}\right)\right]$ of (2.5) is attained at $\nu=\nu_{N}$.

\section{Application to insurance}

Assume that the net premium of the $m$ policyholders is invested in an equity-linked fund whose price follows the dynamics of the Cox-Ross-Rubinstein model, see [4]. Denote the prices by $S_{0}, S_{1}, \ldots, S_{N}$. At time $j=0$ the total investment of the policyholders is $m S_{0}$. In the next time instant the price of the fund is multiplied by $u$ or $d$ with probabilities $p$ and $q=1-p$ respectively with the usual assumptions $d<1<1+r<u$. Many guarantees can be interpreted as contingent claims on the underlying fund. The minimum yield guarantee, to give the simplest example, stipulates that the payment to the policyholder at time $N$ will be equal to at least

$$
G=(1+r)^{N} S_{0}
$$

for some interest rate agreed to in the contract, which we assume to be constant throughout the lifetime of policies. Other types of guarantees can be included as well. If at the expiration the price of the fund reduced by possible fees exceeds $G$ the policyholder gets the bigger of the two sums. If at time $j=0$ the insurer buys $m$ put options on the fund price with strike price $k=(1+r)^{N} S_{0}$ and expiration $N$ that completely offsets the financial risk due to fund price fluctuations. But such a strategy does not take mortality into account. The strategy we will investigate will be a combination of charging fees towards the fund and at an optimal time buy options that at least partially offset financial risks. Paper [2] considers fund linked products with guarantees and an optimal fee structure which means that the insurance company charges a fee towards the underlying fund in an optimal way so that the expected discounted loss for the company is zero. In this paper we will consider a mixed approach. The company will set aside a portion of the fund value as a reserve possibly subject to some conditions. At any time the company can decide to switch to hedging future liabilities with derivatives based on the fund value and the number of surviving policyholders. The fees accumulated will partially offset the cost of the options. We will derive the optimal time to switch which will minimize the expected loss for the company. 
Assume that the $m$ policyholders are of the same age $x$. Denote the number of surviving policyholders at times $j=0,1, \ldots, N$ by $\alpha_{0}, \alpha_{1}, \ldots, \alpha_{N}$. We will assume that mortality is independent of the movement of the fund value. For the sake of simplicity we will consider contracts with no guaranteed minimum death benefit. Note that the sequence $\alpha_{0}, \ldots, \alpha_{N}$ is an inhomogeneous Markov chain due to ageing with $P\left(\alpha_{j+1}=i-k \mid \alpha_{j}=i\right)=$ $\left(\begin{array}{l}i \\ k\end{array}\right) q_{x+j}^{k} p_{x+j}^{i-k}$ for $k=0,1, \ldots, i$ in the usual actuarial notation. For mortality simulation we use [1].

We will apply the theory developed in Section 2 to the Markov chain $\left(S_{j}, \alpha_{j}\right)_{0 \leq j \leq N}$ and the functions $c_{j}$ and $g_{j}$ that we now proceed to identify. We consider the following strategy: The company at each time $j$ either sets aside the difference between the fund price $S_{j}$ and the accumulated value $S_{0}(1+r)^{j}$ if this difference is positive and 0 else, or the company buys a number of put options on the fund at strike price $k=S_{0}(1+r)^{N}$. The number of options to be bought will be determined below in two cases. The options will offset some of the financial risk due to fund price fluctuation but the cost of buying the options will be incurred. In the notation of Section 2 what we set aside will reduce the loss and we define

$$
c_{j}\left(S_{0}, S_{1}, \ldots, S_{j}\right)=-m \max \left(S_{j}-S_{0}(1+r)^{j}, 0\right) .
$$

Let the price of the put option on the price of the fund at strike price $k=S_{0}(1+r)^{N}$ at time $j$ be denoted by $\pi_{j}\left(S_{j}, k, N\right)$ determined in the standard way for the binomial model, see [3]. In our scenario two possible numbers of put options can be considered: the first is to buy $\alpha_{j}$ put options at time $j$ which means that the financial risk is eliminated because the options cover any possible shortfall of the fund price. The insurer will be able to cover liabilities towards surviving policyholders but will incur a cost that will contribute towards the loss. In our notation we put

$$
g_{j}\left(S_{0}, \ldots, S_{j}, \alpha_{0}, \ldots, \alpha_{j}\right)=\alpha_{j} \pi_{j}\left(S_{j}, k, N\right) .
$$

The second possibility is to buy $E\left(\alpha_{N} \mid \alpha_{j}\right)$ options. This only partially offsets the risk of shortfall because there may be more surviving policyhoders than expected. In this case define for $j<N$

$$
g_{j}\left(S_{0}, \ldots, S_{j}, \alpha_{0}, \ldots, \alpha_{j}\right)=E\left(\alpha_{N} \mid \alpha_{j}\right) \pi_{j}\left(S_{j}, k, N\right)
$$

and

$$
g_{N}\left(S_{0}, \ldots, S_{N}, \alpha_{0}, \ldots, \alpha_{N}\right)=\alpha_{N} \cdot \pi_{N}\left(S_{N}, k, N\right) .
$$

In both cases the expression

$$
L_{j}=(1+r)^{-j} g_{j}\left(S_{0}, \ldots, S_{j}, \alpha_{0}, \ldots, \alpha_{j}\right)+\sum_{i=1}^{j-1}(1+r)^{-i} c_{i}\left(S_{0}, \ldots, S_{i}\right)
$$

will be the discounted loss for the company if the option is bought at time $j$. We choose the stopping rule $\nu$ in such a way that the expected loss

$$
E\left(L_{\nu}\right)
$$

will be minimized. Note that the optimality depends on the probabilities $p$ in the underlying model for the fund price. The solution is provided by Theorem 2.3. Note also that $\nu=N$ means that the insurer does not buy options but covers any shortfall from own funds. 
Explicit calculations are not possible so we present two simulations to illustrate the results. The table below defines the parameters used in the simulations:

$\begin{array}{cc}S_{0} & 1 \\ u & 1.04 \\ d & 0.98 \\ r & 0.01 \\ N & 5 \\ m & 1000 \\ x & 30 \\ z & 5000\end{array}$

where $z$ denotes the number of simulations. Table 1 summarizes some statistics for the final loss $L_{N}$ for different $p$ when the insurer buys $\alpha_{\nu}$ options.

Table 1: A few selected descriptive statistics of the final loss distribution when $\alpha_{\nu}$ options are bought.

\begin{tabular}{c|c|c|c|c|c}
$p$ & $E\left(L_{N}\right)$ & $\mathrm{SD}\left(L_{N}\right)$ & 90 th percentile & $E(\nu)$ & $\mathrm{SD}(\nu)$ \\
\hline \hline 0.51 & -32.45 & 135.55 & 87.54 & 4.71 & 0.69 \\
0.50 & -21.08 & 122.52 & 60.58 & 3.70 & 1.57 \\
0.49 & -20.45 & 123.41 & 42.08 & 2.85 & 1.77 \\
0.48 & -17.62 & 117.49 & 41.72 & 2.63 & 1.74
\end{tabular}

Let us now look at results of simulations when the insurer at time $j$ considers buying $E\left(\alpha_{N} \mid \alpha_{j}\right)$ options (Table 2).

Table 2: A few selected descriptive statistics of the final loss distribution when options cover the expected number of surviving policyholders.

\begin{tabular}{c|c|c|c|c|c}
$p$ & $E\left(L_{N}\right)$ & $\mathrm{SD}\left(L_{N}\right)$ & 90 th percentile & $E(\nu)$ & $\mathrm{SD}(\nu)$ \\
\hline \hline 0.51 & 0.69 & 165.27 & 195.57 & 3.45 & 1.60 \\
0.50 & 9.42 & 160.78 & 197.46 & 3.22 & 1.64 \\
0.49 & 11.45 & 158.66 & 196.50 & 3.05 & 1.63 \\
0.48 & 18.36 & 152.96 & 197.24 & 2.75 & 1.64
\end{tabular}

In the first case the loss is negative and the financial risk is completely offset. It is true that such a strategy depends on assumptions about availability of derivatives but in more realistic settings it can still be used to reduce the cost of guarantees. In the second case note that the loss due to $\alpha_{T}$ exceeding the expected number of survivors needs to be taken into account because it contributes to the overall loss.

For the case $p=0.48$ the distribution of final loss $L_{N}$ and optimal stopping time $\nu$ are shown in Figure 1 for both numbers of options.

\section{Conclusions}

We propose a strategy of hedging liabilities arising from equity-linked products with minimum guarantees with financial derivatives. Simulations show that there is an optimal time 


\section{Case 1}
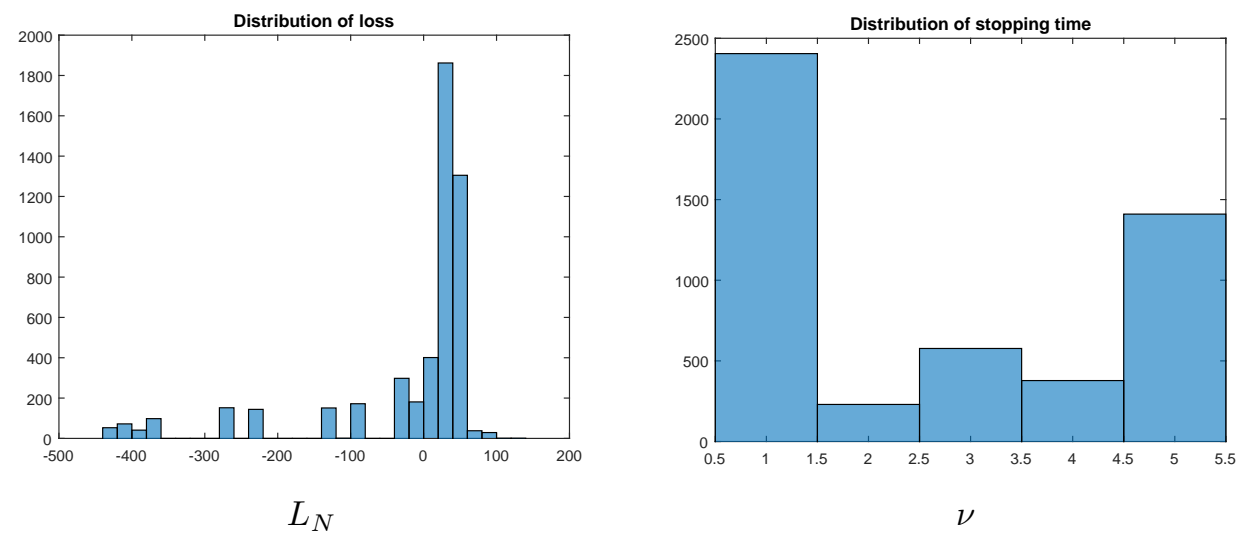

\section{Case 2}
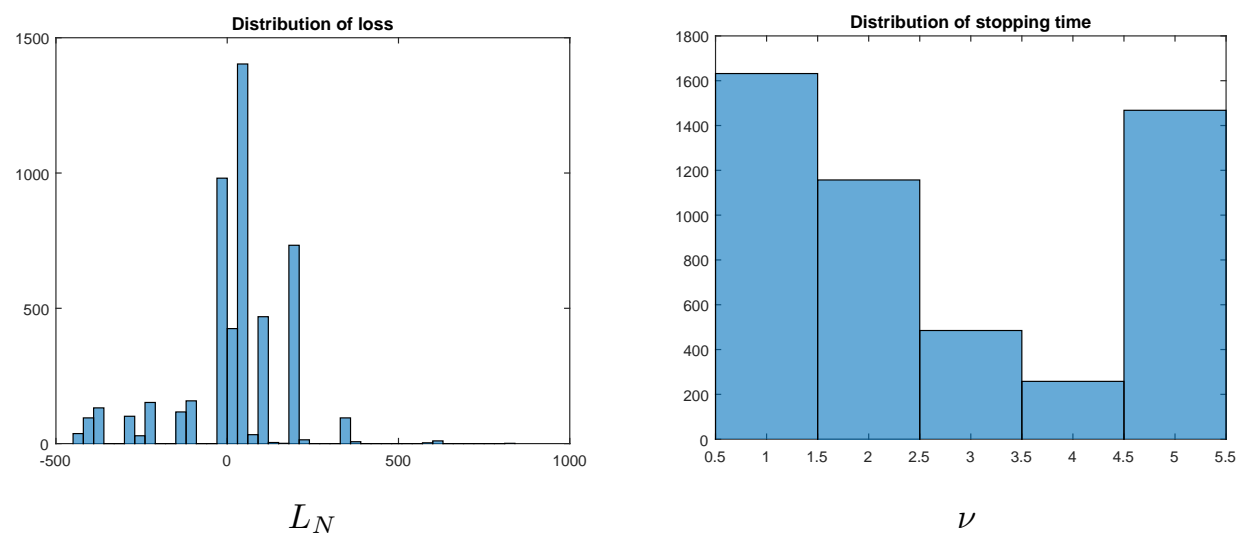

Figure 1: The distribution of final loss and optimal time $\nu$.

to switch from charging a fee towards the fund to buying a put option that will offset the financial risk. The cases considered are simplified but may be an indication that strategies for more realistic settings are possible.

\section{References}

[1] Slovenian mortality tables SIA65, 2010 (Slovenske rentne tablice smrtnosti), Official Gazette of the Republic of Slovenia 26 (2016), 2148-2149, https: //www.uradni-list.si/ _pdf/2016/Ur/u2016018.pdf.

[2] A. R. Bacinello, P. Millossovich, A. Olivieri and E. Pitacco, Variable annuities: a unifying valuation approach, Insurance Math. Econom. 49 (2011), 285-297, doi:10.1016/j.insmatheco. 2011.05.003.

[3] T. Björk, Arbitrage Theory in Continuous Time, Oxford Finance Series, Oxford University Press, 3rd edition, 2009. 
[4] J. C. Cox, S. A. Ross and M. Rubinstein, Option pricing: a simplified approach, J. Financial Econ. 7 (1979), 229-263, doi:10.1016/0304-405x(79)90015-1.

[5] J. Dhaene, A. Kukush, E. Luciano, W. Schoutens and B. Stassen, On the (in-)dependence between financial and actuarial risks, Insurance Math. Econom. 52 (2013), 522-531, doi:10. 1016/j.insmatheco.2013.03.003.

[6] T. Møller, Hedging equity-linked life insurance contracts, N. Am. Actuar. J. 5 (2001), 79-95, doi:10.1080/10920277.2001.10595986.

[7] D. J. F. Nonnenmacher, Guaranteed equity-linked products, in: Proceedings of the 8th International AFIR Colloquium, 1998 pp. 413-428, held in Robinson College at Cambridge University, UK, September 15 - 17, 1998, http://www.actuaries.org/AFIR/colloquia/ Cambridge/Nonnenmacher.pdf.

[8] D. J. F. Nonnenmacher and J. Ruß, Equity-linked life insurance in Germany: quantifying the risk of additional policy reserves, in: Proceedings of the 7th International AFIR Colloquium, Volume 2, 1997 pp. 719-738, held in the Cairns International Hotel, North Queensland, Australia, August 11 - 15, 1997, http://www.actuaries.org/AFIR/colloquia/ Cairns/Nonnenmacher_Russ.pdf.

[9] A. N. Shiryayev, Optimal Stopping Rules, volume 8 of Stochastic Modelling and Applied Probability, Springer-Verlag, New York, 2008, doi:10.1007/978-3-540-74011-7, translated from Russian by A. B. Aries (original Russian edition published by Nauka, Moscow, 1976).

[10] B. Zgrablić, The equalization scheme of the residual voluntary health insurance in Slovenia, Ars Math. Contemp. 8 (2015), 225-234, doi:10.26493/1855-3974.667.fec. 